\title{
Comunicação
}

[Communication]

\section{Ocorrência de Contracaecum pelagicum Johnston \& Mawson, 1942 (Nematoda, Anisakidae), em pinguim-de-magalhães (Spheniscus magellanicus Forster, 1781) (Aves, Spheniscidae) no litoral do Espírito Santo}

[Occurrence of Contracaecum pelagicum Johnston \& Mawson, 1942 (Nematoda, Anisakidae) in Magellanic penguin (Spheniscus magellanicus Forster, 1781) (Aves, Spheniscidae) at Espirito Santo coast]

\author{
N.B. Ederli ${ }^{1,2}$, F.C.R. Oliveira ${ }^{2 *}$, C.M. Monteiro ${ }^{3}$, L.S. Silveira ${ }^{4}$, M.L.A. Rodrigues ${ }^{1}$ \\ ${ }^{1}$ Instituto de Veterinária - UFRRJ - Seropédica, RJ \\ ${ }^{2}$ Centro de Ciência e Tecnologias Agropecuárias - UENF \\ Av. Alberto Lamego, 2000 \\ 28013-800 - Campos dos Goitacazes, RJ \\ ${ }^{3}$ Instituto de Biociências - UFRRJ - Seropédica, RJ \\ ${ }^{4}$ Núcleo de Pesquisa em Animais Silvestres - UENF - Campos dos Goitacazes, RJ
}

Existem 17 espécies de pinguins, sendo o pinguim-de-magalhães (Spheniscus magellanicus Forster, 1781) a espécie de maior ocorrência na costa brasileira, de onde chegam carregados pelas correntes das Malvinas (Valim et al., 2004). É comum encontrar estas aves debilitadas ou mortas no litoral devido ao esgotamento de suas reservas ou pela contaminação por óleo. Sua dieta é constituída basicamente de peixes (Frere et al., 1996). Uma variada fauna de helmintos pode ser encontrada parasitando as aves, incluindo nematoides, digenéticos, cestoides e acontocéfalos (Pazos et al., 2003).

Contracaecum pelagicum Johnston \& Mawson, 1942, é um nematoide pertencente à família Anisakidae. É hospedeiro intermediário e paratênico de invertebrados aquáticos e peixes, respectivamente. Os hospedeiros definitivos das espécies do gênero Contracaecum Railliet \& Henry, 1912, são aves e mamíferos piscívoros (Anderson, 2000). Contracaecum pelagicum foi registrado parasitando albatroz Diomedea melanophris (Temminck, 1828) atualmente chamada de Thalassarche melapophris (Temminck, 1828), atobá-marrom (Sula leucogaster Boddaert, 1783) e pinguim-demagalhães (S. magellanicus) (Garbin et al., 2007).
Este trabalho teve como objetivo relatar a ocorrência de nematoides gastrintestinais em um pinguim-de-magalhães encontrado morto no litoral de Vitória, no Estado do Espírito Santo, Brasil.

Durante a necropsia de um pinguim-demagalhães, o conteúdo estomacal foi colhido, conservado em formalina $10 \%$ e encaminhado ao Laboratório de Sanidade Animal, do Centro de Ciências e Tecnologias Agropecuárias da Universidade Estadual do Norte Fluminense Darcy Ribeiro. Os nematoides presentes foram recolhidos, fixados em AFA e, posteriormente, transferidos para etanol $70 \%$ glicerinado (etanol 9: glicerina 1). Os parasitos foram clarificados em lactofenol de Amann e montados entre lâmina e lamínula em montagem temporária. Os nematoides foram identificados, quantificados e mensurados utilizando-se microscópio óptico Zeiss - Axiostar Plus com câmera digital CanonPowerShot A640 para captura de imagens e Software Zeiss AxionVision Sample Images para análise de imagens. As medidas foram dadas em micrômetros.

O número e a distribuição das papilas précloacais (28-31) e pós-cloacais (7) de seis espécimes machos (Fig. 1) de C. pelagicum

Recebido em 11 de julho de 2008

Aceito em 12 de maio de 2009

*Autor para correspondência (corresponding author)

E-mail: folieira@uenf.br 
observados são semelhantes a relatos anteriores para essa espécie (Garbin et al., 2007). Estas foram as principais características usadas no diagnóstico na determinação da espécie. Outras características, como distribuição das papilas labiais, relação ceco intestinal/apêndice ventricular 2,48 a 3,58:1 e tamanho dos espículos em relação ao comprimento do corpo 21,42 $(20,41-24,78)$ também estão dentro da variação conhecida para a espécie e serviram como complementação para a identificação da espécie
(Johnson e Mawson, 1942; Lent e Teixeira de Freitas, 1948; Santos, 1984; Vicente et al., 1995; Garbin et al., 2007).

Foram encontrados grande número de espécimes juvenis e 48 adultos, sendo 24 machos e 24 fêmeas (Fig. 2). A presença de espécimes juvenis permitiu inferir que o pinguim-de-magalhães ingeria constantemente hospedeiros intermediários, peixes, infectados por larvas de C. pelagicum.

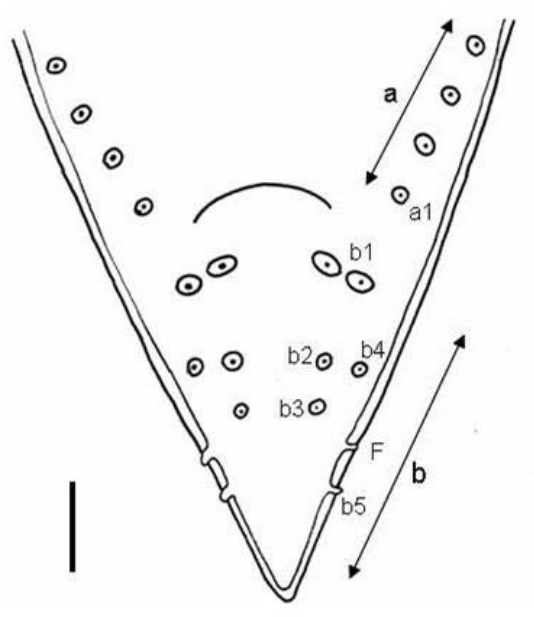

Figura 1. Contracaecum pelagicum Johnston \& Mawson, 1942, de pinguim-de-magalhães, Spheniscus magellanicus Forster, 1781. Extremidade posterior de um macho em vista ventral: (a) papilas pré-cloacais e (a1) papila proximal; (b) papilas pós-cloacais e (b1) par de paracloacais papilas; (b2-b3) papilas subventrais; (b4-b5) papilas sublaterais; (F) fasmídeos; barra $=50 \mu \mathrm{m}$.
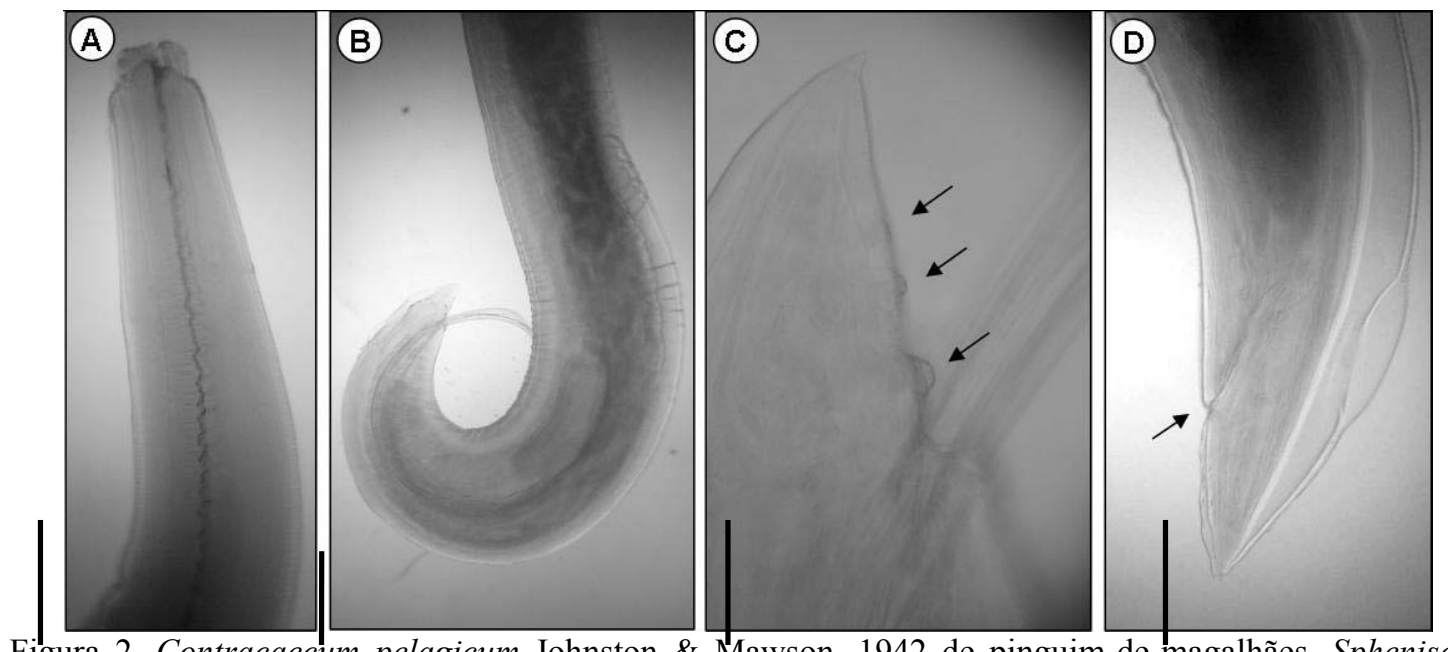

Figura 2. Contracaecum pelagicum Johnston \& Mawson, 1942 de pinguim-de-magalhães, Spheniscus magellanicus Forster, 1781. Espécimes adultos: (A) extremidade anterior de uma fêmea, barra $=250 \mu \mathrm{m}$; (B) extremidade posterior de um macho, barra $=500 \mu \mathrm{m}$; $(\mathrm{C})$ ponta da cauda de um macho: setas evidenciam papilas, barra $=100 \mu \mathrm{m}$; (D) extremidade posterior de uma fêmea: seta evidencia abertura do ânus, barra $=250 \mu \mathrm{m}$. 
A presença de pinguins é relativamente comum no litoral da região Sudeste do Brasil. Eles são capturados em redes de pesca ou encontrados debilitados ou mortos (Frere et al., 1996). Aparentemente, não há pesquisas que tenham identificado a verdadeira causa de morte desses animais, ou que tenham estudado o efeito do parasitismo sobre o déficit energético das aves capturadas. Este trabalho é o primeiro relato do parasitismo por $C$. pelagicum em pinguim-demagalhães encontrado no Sudeste do Brasil.

Palavras-chave: pinguim-de-magalhães, Spheniscus magellanicus, Nematoda, Contracaecum pelagicum

\begin{abstract}
The occurrence of gastrointestinal nematodes affecting a Magellanic penguin, which was submitted to necropsy after being found dead at the coast of Vitória, Espirito Santo State, Brazil, was reported. A great number of juveniles nematodes and 48 adults (24 males and 24 females) were found in the stomach of the penguin. The parasite was diagnosed as Contracaecum pelagicum in agreement with the number and the distribution of the precloacal and postcloacal papillae of the male specimens. Other characteristics as distribution of the labial papillae, intestinal caecum and ventricular appendix ratio, spicules length, and total body length ratio were also in accordance to the well-known variation for the species, confirming the diagnosis. This work is the first report of the parasitism by $\mathrm{C}$. pelagicum in Magellanic penguin found in the Southeast of Brazil.
\end{abstract}

Keywords: Magellanic penguin, Spheniscus magellanicus, Nematoda, Contracaecum pelagicum

\section{REFERÊNCIAS BIBLIOGRÁFICAS}

ANDERSON, R.C. Nematode parasites of vertebrates: their development and transmission. 2.ed. Wallingford, UK: CAB International, 2000. $650 \mathrm{p}$.

FRERE, E.; GANDINI, P.; LICHTSCHEIN, V. Variación latitudinal en la dieta del pinguino de Magallanes (Spheniscus magellanicus) em la costa patagónica, Argentina. Ornitol. Neotrop., v.7, p.35-41, 1996.

GARBIN, L.E.; NAVONE, G.T.; DIAZ, J.I. et al. Further study of Contracaecum pelagicum (Nematoda: Anisakidae) in Spheniscus magellanicus (Aves: Spheniscidae) from Argentinean coasts. J. Parasitol., v.93, p.143150, 2007.

JOHNSTON, T.H.; MAWSON, P.M. Nematodes from Australian albatrosses and petrels. Trans. $R$. Soc. S. Aust., v.66, p.66-70, 1942.

LENT, H.; TEIXEIRA DE FREITAS, J.F. Uma coleção de nematódeos, parasitos de vertebrados, do Museu de História Natural de Montevideo. Mem. Inst. Oswaldo Cruz, v.46, p.1-71, 1948.
PAZOS, G.E.; LAURENTI, S.; DÍAZ, J.I. Helmintofauna del pinguino de Magallanes (Spheniscus magellanicus) em península Valdes, província del Chubut. Resultados preliminares. Hist. Nat., v.2, p.85-94, 2003.

SANTOS, C.P. Um nematódeo parasita do pinguim Spheniscus magellanicus (Forster) (Ascaridoidea, Anisakidae). Mem. Inst. Oswaldo Cruz, v.79, p.233-237, 1984.

VALIM, M.P.; SILVA, L.H.M.; AMORIM, M. et al. Encontro de Spheniscus magellanicus (Forster, 1781) (Aves: Sphenisciformes) parasitado por Austrogoniodes bifasciatus (Piaget, 1885) (Ischnocera: Philopteridae) na Região dos Lagos, Estado do Rio de Janeiro, Brasil. Entomol. Vect., v.11, p.191-194, 2004.

VICENTE, J.J.; RODRIGUES, H.O.; GOMES, D.C. et al. Nematoides do Brasil. Parte IV. Nematoides de aves. Rev. Bras. Zool., v.12, supl.7, p.1-273, 1995. 